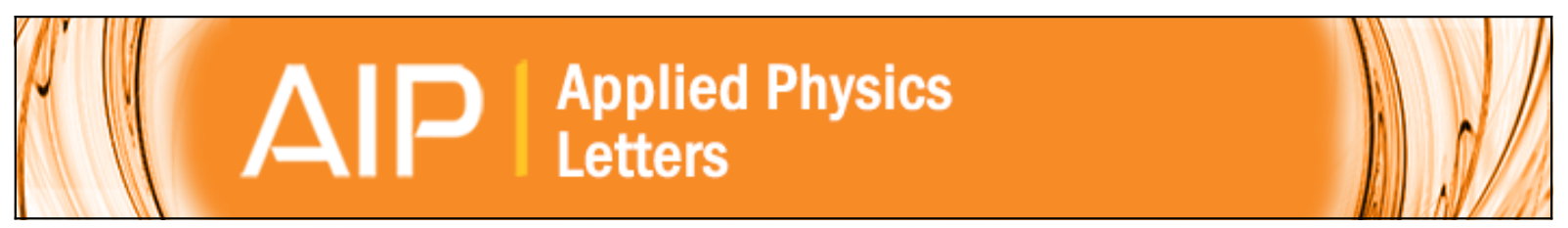

\title{
Tapered silicon nanowires for enhanced nanomechanical sensing
}

O. Malvar, E. Gil-Santos, J. J. Ruz, D. Ramos, V. Pini, M. Fernandez-Regulez, M. Calleja, J. Tamayo, and A. San Paulo

Citation: Applied Physics Letters 103, 033101 (2013); doi: 10.1063/1.4813819

View online: http://dx.doi.org/10.1063/1.4813819

View Table of Contents: http://scitation.aip.org/content/aip/journal/apl/103/3?ver=pdfcov

Published by the AIP Publishing

\section{Articles you may be interested in}

Silicon nanowire arrays coated with electroless Ag for increased surface-enhanced Raman scattering APL Mater. 3, 056101 (2015); 10.1063/1.4921040

Photoresponsive properties of ultrathin silicon nanowires Appl. Phys. Lett. 105, 231116 (2014); 10.1063/1.4904089

Molecular doping and gas sensing in Si nanowires: From charge injection to reduced dielectric mismatch J. Appl. Phys. 114, 204302 (2013); 10.1063/1.4834576

Silicon nanowire based Pirani sensor for vacuum measurements Appl. Phys. Lett. 101, 183506 (2012); 10.1063/1.4765665

Piezoresistive silicon nanowire based nanoelectromechanical system cantilever air flow sensor Appl. Phys. Lett. 100, 023111 (2012); 10.1063/1.3675878

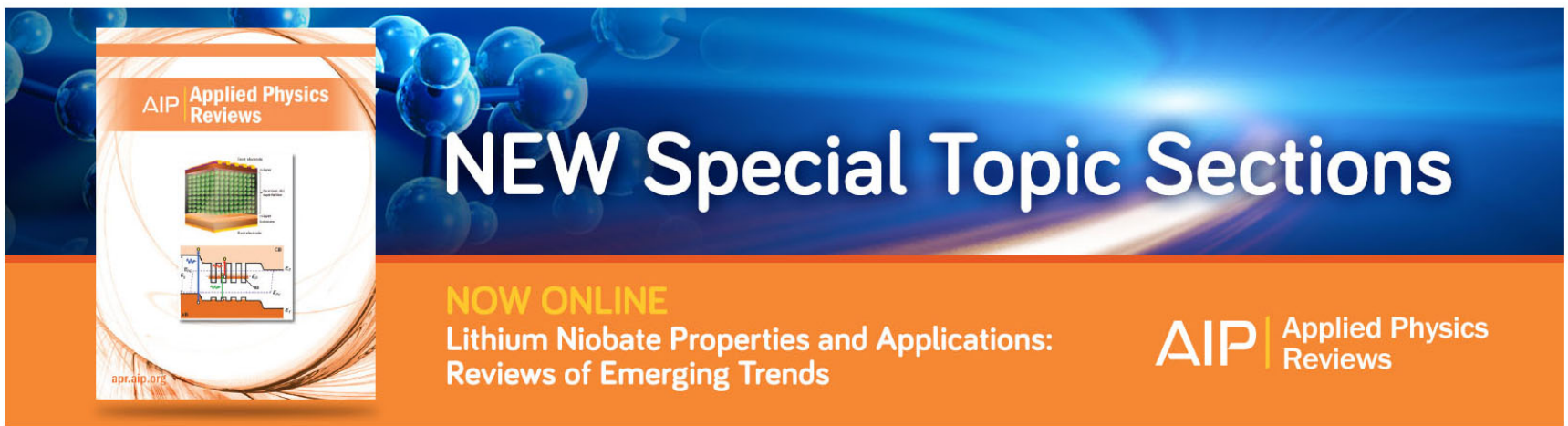




\title{
Tapered silicon nanowires for enhanced nanomechanical sensing
}

\author{
O. Malvar, ${ }^{1}$ E. Gil-Santos, ${ }^{1}$ J. J. Ruz, ${ }^{1}$ D. Ramos, ${ }^{2}$ V. Pini, ${ }^{1}$ M. Fernandez-Regulez, ${ }^{3}$ \\ M. Calleja, ${ }^{1}$ J. Tamayo, ${ }^{1}$ and A. San Paulo ${ }^{1, a)}$ \\ ${ }_{1}^{1}$ Instituto de Microelectrónica de Madrid, IMM-CNM (CSIC), 28760 Tres Cantos, Spain \\ ${ }^{2}$ School of Engineering and Applied Sciences, Harvard University, Cambridge, Massachusetts 02138, USA \\ ${ }^{3}$ Instituto de Microelectrónica de Barcelona, IMB-CNM (CSIC), Campus UAB, 08193 Bellaterra, Spain
}

(Received 18 April 2013; accepted 26 June 2013; published online 15 July 2013)

\begin{abstract}
We investigate the effect of controllably induced tapering on the resonant vibrations and sensing performance of silicon nanowires. Simple analytical expressions for the resonance frequencies of the first two flexural modes as a function of the tapering degree are presented. Experimental measurements of the resonance frequencies of singly clamped nanowires are compared with the theory. Our model is valid for any nanostructure with tapered geometry, and it predicts a reduction beyond two orders of magnitude of the mass detection limit for conical resonators as compared to uniform beams with the same length and diameter at the clamp. (C) 2013 AIP Publishing LLC. [http://dx.doi.org/10.1063/1.4813819]
\end{abstract}

Resonant beams with their thickness or diameter in the nanometer scale are essential components of nanoelectromechanical systems (NEMS). ${ }^{1}$ The singular properties of bottom-up synthesized nanostructures, such as Si nanowires (NWs) obtained by vapor-liquid-solid (VLS) growth, ${ }^{2}$ offer unique opportunities to investigate on NEMS functionalities. In particular, the exceptional performance of VLS Si NW resonators $^{3-5}$ has been demonstrated to provide unprecedented functional features as nanomechanical sensors, such as highly efficient transduction mechanisms, ${ }^{6-9}$ ultrasensitive physical sensing, ${ }^{10,11}$ and complex actuation schemes such as self-excitation ${ }^{12}$ or parametric amplification. ${ }^{13}$ One particular singular feature of Si NWs is that the VLS growth mechanism can produce a tapered cross-section. Tapered Si NWs have, indeed, been usually considered undesirable for most applications, and many efforts regarding growth optimization have focused on obtaining uniform NWs with negligible diameter variation. However, this effect offers exceptional opportunities for obtaining devices with matchless performance characteristics. For instance, excellent field-emission characteristics have been attributed to $\mathrm{Si}$ NWs with taper-like geometry; ${ }^{14}$ conically shaped Si NWs produced with engineered apex angles have been suggested for rational design of functional nanostructures with control of the spectral and spatial electromagnetic scattering signatures; ${ }^{15}$ also, the enhanced light absorption observed in vertical arrays of $\mathrm{Si}$ nanocones has revealed a promising approach to enhance the solar cell energy conversion efficiency in photovoltaic applications. ${ }^{16}$

In the context of nanomechanical devices, the flexural vibration modes of beams with a tapered geometry present significantly different resonance frequencies, mode shapes, and effective masses as compared to uniform beams. These differences have been widely neglected so far in the development of nanomechanical devices based on NWs, thus limiting their truthful practical application. In this work, we analyze the effect of a taper-like geometry on the mechanical

\footnotetext{
${ }^{\text {a) }}$ Author to whom correspondence should be addressed. Electronic mail: alvaro.sanpaulo@csic.es
}

resonances of singly clamped beams based on Si NWs. We present experimental measurements of the resonance frequencies of the first and second order flexural modes of tapered Si NWs that do not match elastic beam theory for a uniform cross-section. Then we provide a theoretical model that accounts for the tapered geometry so that it reproduces the experimental results. Our model is further validated by finite element method (FEM) simulation and analysis of the mode shape profiles and then used to discuss the performance of tapered resonators as nanomechanical mass sensors.

The Si NWs used in this study were grown via the VLS mechanism in a chemical vapor deposition system using $\mathrm{SiCl}_{4}$ as precursor. Au colloidal nanoparticles (NP) were used as metal catalyst. In VLS growth, the NP diameter determines the NW diameter at the base. Then, tapering during growth occurs via two possible mechanisms: on one hand, gradual size reduction of the catalyst NP during growth by either diffusion, evaporation, or chemical reactions results in a time-varying NW diameter; ${ }^{17}$ on the other hand, dissociative adsorption of the precursor gas on the gas/solid interface produces a progressive increase of the NW diameter. ${ }^{18}$ The simultaneous presence of both effects is possible, ${ }^{19}$ and either mechanism dominates depending on the growth conditions. Precise control of the growth conditions has indeed been demonstrated to enable either extremely long NWs with uniform diameter ${ }^{20}$ or tapered NWs with precise control of their geometry. ${ }^{21} \mathrm{~A}$ recent study demonstrates that accurate tapering control can be achieved by proper setting of the $\mathrm{SiCl}_{4} / \mathrm{H}_{2}$ molar ratio, because this factor governs the interplay between the catalyzed VLS growth at the NW end and the uncatalyzed Si deposition at the NW sidewalls. ${ }^{21}$ In our case, slight variations in the carrier gas flow rate were introduced in order to obtain NWs with different degree of tapering (see supplemental material ${ }^{32}$ ). The NWs were horizontally grown at the vertical sidewalls of prefabricated $\mathrm{Si}$ microstructures in a silicon-on-insulator (SOI) substrate [Figs. 1(a) and 1(b)]. ${ }^{22}$ The NWs' length $L$ ranges from 5 to $25 \mu \mathrm{m}$, and their radius at the base $R_{0}$ ranges from 40 to $150 \mathrm{~nm}$. The distance between the NWs and the substrate $d_{S}$ lies in the $1-2 \mu \mathrm{m}$ range. The experimental measurements of 
the NW resonance frequencies were performed with a homemade interferometry system as described elsewhere. ${ }^{10}$

Figures 1(c) and 1(d) show a representative example of the measured thermomechanical spectra of a tapered NW around the resonance frequencies of the first and second flexural modes, respectively. The two peaks observed for each mode are the result of two orthogonal vibrations of the NW in perpendicular planes. This mode splitting effect can occur in any nanowire-like beam resonator, regardless of having a tapered or a uniform geometry, and it is a consequence of structural defects such small asymmetries in the crosssection or the clamp. ${ }^{23}$ The peak separation is very similar for both modes, i.e., $0.57 \%$ for the first mode and $0.51 \%$ for the second mode. Using the higher amplitude peak as a reference, we find that the ratio between the resonance frequencies $f_{1}$ and $f_{2}$ of the first and second modes is far from the expected value of $f_{2} / f_{1}=6.267$ for beams with uniform section. $^{24}$ For the case shown in Figure 1, we obtain $f_{2} / f_{1}=3.960$.

We describe the tapering degree by a parameter $\alpha$ that we define as
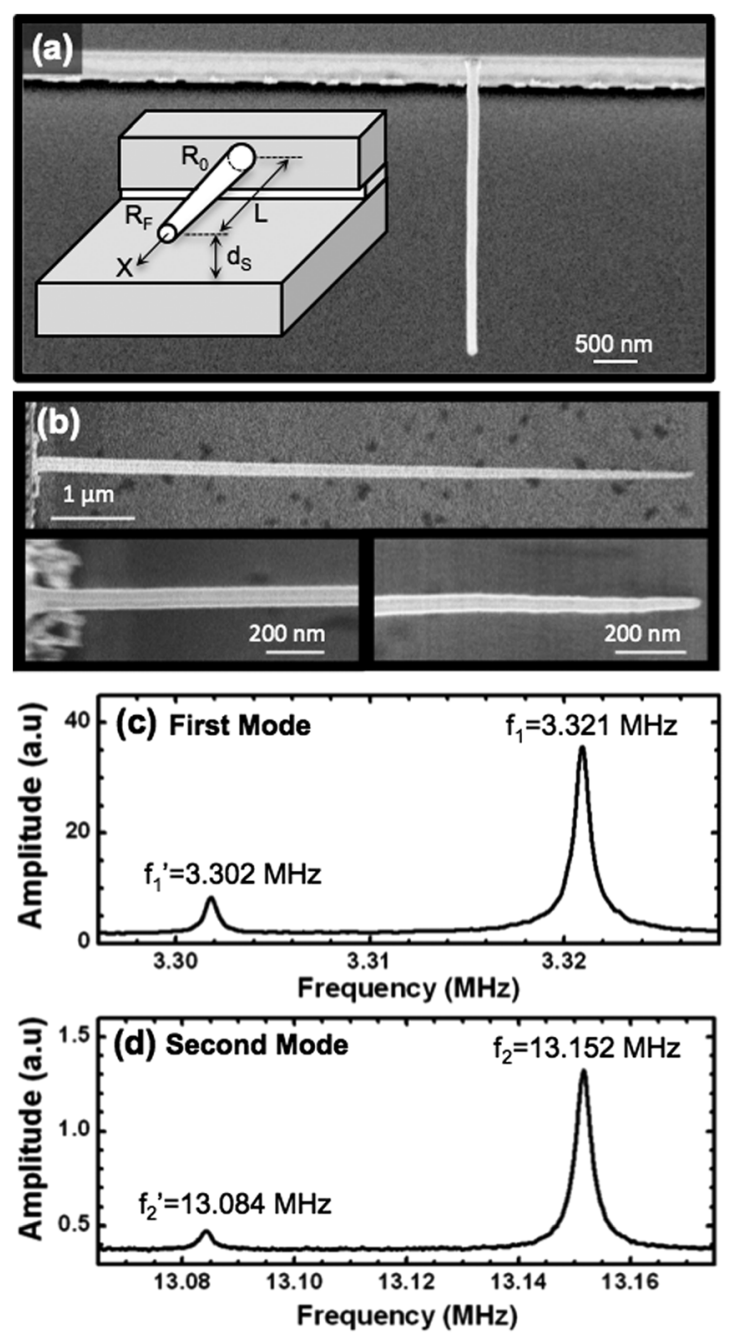

FIG. 1. (a) SEM image and schematic diagram showing the location of a NW at the vertical sidewall of a step fabricated in a SOI substrate. (b) SEM image of a highly tapered NW with a length of $7.5 \mu \mathrm{m}$, a radius of $50 \mathrm{~nm}$ at the clamp, and a radius of $20 \mathrm{~nm}$ at the free end. (c), (d) Measured mechanical resonances of a tapered NW around the first and second modes, respectively.

$$
\alpha=\frac{R_{0}-R_{F}}{R_{0}}
$$

where $R_{0}$ and $R_{F}$ are the values of the NW radius at the clamp and at the free-end, respectively. Therefore, $\alpha=0$ corresponds to a uniform cross-section and $\alpha=1$ represents a conical NW. Fig. 2 summarizes several measurements of $f_{1}$ and $f_{2}$ obtained for NWs with different degree of tapering. The tapering parameter was determined from SEM measurements. In Fig. 2(a), we plot the experimental values of $f_{1}$ and $f_{2}$ divided by the theoretical values for a uniform crosssection, $f_{U 1}$ and $f_{U 2}$. These were calculated from EulerBernoulli theory for NWs with the same length and a uniform cross-section with a radius corresponding to the measured radius at the clamp, according to the expression

$$
f_{U n}=\frac{1}{2 \pi}\left(\frac{k_{n}}{L}\right)^{2} \sqrt{\frac{E I_{0}}{\rho S_{0}}},
$$

where $n$ indicates the mode order, $L$ is the length, $E$ is the Young's modulus, $\rho$ is the density, $I_{0}$ is the second moment of area, and $S_{0}$ is the cross-section area. We consider a circular cross-section so that $S_{0}=\pi R_{0}{ }^{2}$ and $I_{0}=\pi R_{0}^{4} / 4$. The parameter $k_{n}$ depends on the mode order, and it takes the values $k_{1}=1.875$ and $k_{2}=4.694$. Fig. 2(a) shows that for $\alpha$ close to 0 , the experimental values of $f_{1}$ and $f_{2}$ approach the theoretical values for a uniform cross-section, so that both ratios $f_{1} / f_{U 1}$ and $f_{2} / f_{U 2}$ are close to 1 . However, for increasing $\alpha, f_{1} / f_{U 1}$ increases and reaches values around 1.5 for $\alpha$ around 0.65 , whereas $f_{2} / f_{U 2}$ decreases to values around 0.9. Fig. 2(b) displays the experimental values of the ratio $f_{2} / f_{1}$ for different NWs as a function of $\alpha$. The ratio $f_{2} / f_{1}$ differs increasingly from the theoretical value of 6.267 for increasing $\alpha$.

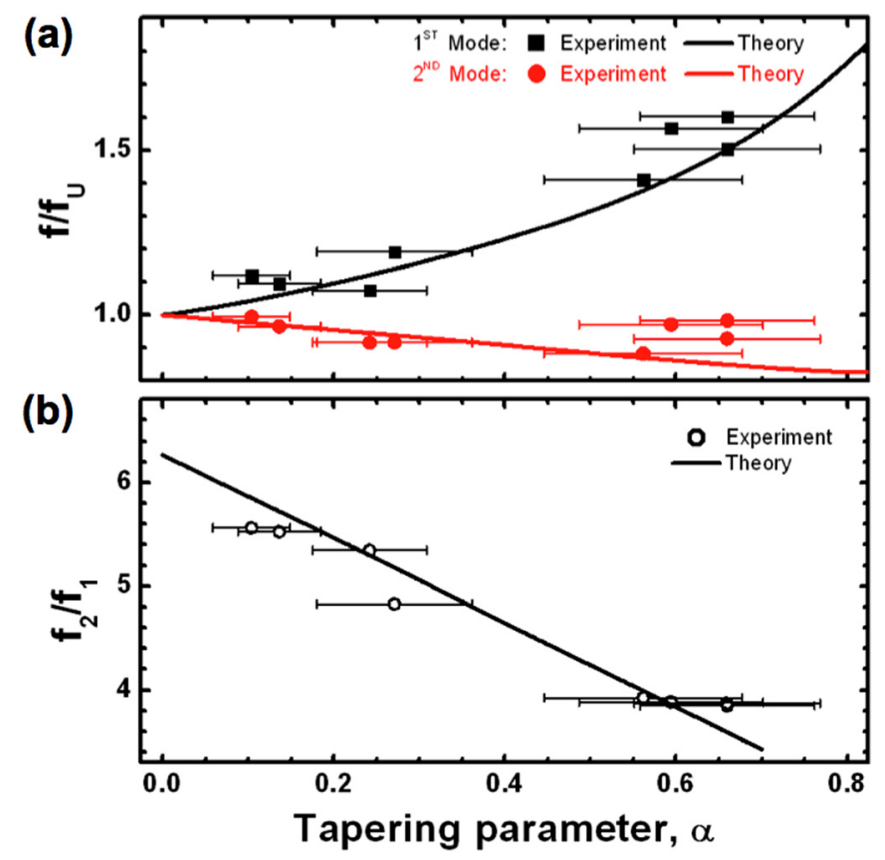

FIG. 2. (a) Ratio of the resonance frequencies of tapered Si NWs to that of uniform ones with the same length and radius at the base vs. the tapering parameter $\alpha$ (symbols: experimental data; lines: analytical theory). (b) Ratio of the resonance frequencies of the first and second modes vs. the tapering parameter $\alpha$ (symbols, experimental data; line, analytical theory). 
In order to provide a theoretical description of tapered NW vibrations we have solved the Euler-Bernoulli equation for the general case of beams with non-uniform cross-section ${ }^{25}$

$$
\rho S(x) \frac{\partial^{2} w_{n}(x, t)}{\partial t^{2}}+\frac{\partial^{2}}{\partial x^{2}}\left[\frac{E I(x)}{L^{4}} \frac{\partial^{2} w_{n}(x, t)}{\partial x^{2}}\right]=0,
$$

where $w_{n}$ is the $n$th order mode contribution to the beam displacement at the dimensionless (normalized to length) longitudinal position $x$ and time $t$. The cross-section area $S(x)$ and area moment $I(x)$ depend on the position $x$ in order to account for the tapered geometry. The solution to Eq. (3) can be expressed in terms of Bessel functions (see supplemental material ${ }^{32}$ ), and the resonance frequencies can be written in terms of a parameter $k_{n}$ that now depends on the tapering degree $\alpha$

$$
f_{n}=\frac{1}{2 \pi}\left[\frac{k_{n}(\alpha)}{L}\right]^{2} \sqrt{\frac{E I_{0}}{\rho S_{0}}} .
$$

The application of the corresponding boundary conditions for a singly clamped beam leads to calculate the resonance frequencies $f_{n}$. Unfortunately, it is not possible to reach an exact analytical expression for $k_{n}(\alpha)$, and the resonance frequencies have to be determined numerically. However, we can provide a good approximation of a simple form if we write $k_{n}(\alpha)$ as

$$
k_{n}(\alpha) \approx \sum_{i} a_{n, i} \cdot \alpha^{i}
$$

The coefficients $a_{n, i}$ can then be obtained by numerical fitting to the exact numerical solutions (see supplemental material $^{32}$ ). Figure 2(a) represents (lines) the solutions given by the analytical approximation for $f_{1}$ and $f_{2}$, divided by the expected theoretical values for a uniform cross-section, $f_{U 1}$ and $f_{U 2}$. The graph shows a noticeable good agreement between the experimental measurements and the theoretical calculations.

We can also calculate the ratio $f_{2} / f_{1}$ as a function of $\alpha$. The result can be accurately approximated in the range $0 \leq \alpha \leq 0.9$ by the simple expression (see supplemental material $^{32}$ )

$$
\frac{f_{2}}{f_{1}} \approx 6.267-4.103 \alpha
$$

This result is compared in Fig. 2(b) to the experimental values of the ratio $f_{2} / f_{1}$. The agreement is clearly noticeable. Remarkably, this result implies that the effect of tapering on the ratio $f_{2} / f_{1}$ is independent on the NW length and material properties, and it only depends on the amount of tapering as given by the parameter $\alpha$. Figure 2(b) also suggests that this expression could be used to determine the tapering parameter from the measurement of $f_{2} / f_{1}$ without the need of highresolution electron microscopy imaging.

The application of boundary conditions also allows to analyze the effects of tapering on the mode shape and curvature profiles. This is shown, respectively, in Figures 3(a) and 3(c) for the first mode and in Figs. 3(b) and 3(d) for the second mode. The profiles were obtained for different values of
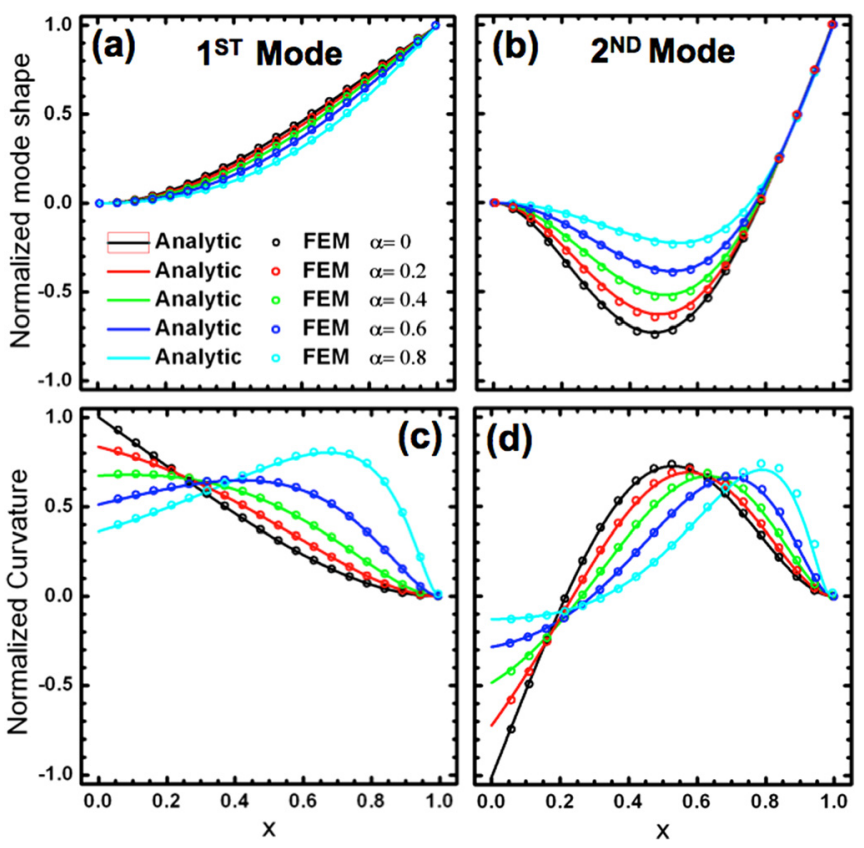

FIG. 3. (a) and (b) Normalized mode shape profiles of the first and second order modes for NWs with different tapering degree (symbols: finite element simulations; lines: analytical model; each curve normalized to maximum displacement for each value of $\alpha$ ). (c), (d) Curvature profiles of the first and second order modes for NWs with varying amount of tapering (symbols: finite element simulations; lines: analytical model; each curve normalized to maximum curvature for $\alpha=0$ ).

the tapering parameter $\alpha$, and the results from the analytical model are compared to FEM (COMSOL) simulations. The agreement is remarkable in all cases. These calculations also provide significant information about the effects of tapering on the NW vibration. First, the mode shape for both modes shows that the vibration is localized closer to the free end as tapering increases. Second, the curvature of the first mode at the clamp is significantly reduced as tapering increases, and the point of maximum curvature moves from the clamp (at $x=0$ for $\alpha=0$ ) to an intermediate position (at $x \approx 0.7$ for $\alpha=0.8$ ); for the second mode, the curvature at the clamp is also reduced for increasing tapering, and the point of maximum curvature (at $x \approx 0.5$ for $\alpha=0$ ) also moves closer to the free end (at $x \approx 0.8$ for $\alpha=0.8$ ).

The results presented in Figure 3 demonstrate important implications of tapering for the application of resonant NWs as nanomechanical sensors. A resonant nanobeam can be used as a nanomechanical mass sensor by tracking the changes in its resonance frequencies as a consequence of the adsorption of sample material. ${ }^{26-30}$ Both the mass and elasticity (Young's modulus) of adsorbed material can be sensed in this manner. ${ }^{10}$ The point along the NW of maximum mass sensitivity is that of maximum vibration amplitude, whereas the point of maximum sensitivity to elasticity corresponds to that of maximum curvature. Considering the mode shape and curvature changes induced by tapering, the point of maximum mass sensitivity remains at the free end for both modes, while the point of maximum sensitivity to elasticity moves from the clamp to forward positions for the first mode, and from the middle to also forward positions for the second mode. In all cases, a consequence of tapering is that the overall dependence of the sensitivity to both mass and elasticity on the 
position along the NW suffers important variations that should be considered for an accurate determination of these magnitudes from resonance frequency shift measurements.

We have analyzed the particular effect of tapering on the mass sensitivity of Si NW resonators at the free end. The minimum detectable mass change $\delta M$ of the system is given by ${ }^{31}$

$$
\delta M=-\frac{2 m_{n}}{f_{n}} \delta f=\mathcal{R}_{n}^{-1} \delta f,
$$

where $m_{n}$ is the effective mass of the $n$th mode and $\delta f$ is the minimum detectable frequency shift. $\mathcal{R}_{n}$ is referred to as responsivity. According to this expression, the minimum detectable mass change with a tapered NW will differ from that of a uniform NW due to both the changes in resonance frequencies and effective masses of the resonant modes. In order to obtain the effective masses of each mode for a tapered NW we apply the Rayleight method

$$
m_{n}=\int_{0}^{1} \rho S(x) w_{n}(x)^{2} d x .
$$

The effect of tapering on the effective mass is actually two-fold: on one hand, it modifies the inertial mass $M$ by the reduction of volume produced by the non-uniform crosssection; on the other hand, the modification of the modeshape profile provides an additional and actually larger reduction of the effective mass. Figure 4(a) shows the calculation of the effective masses of the first two order modes. In order to compare with the case of uniform cross-section, the graph represents the ratio of the effective mass of the first two modes of a tapered NW to that of a uniform NW with the same length and same radius at the clamp, as a function of the tapering parameter $\alpha$. The figure also displays the ratio of the inertial mass of a tapered NW to that of a uniform one with the same length and same radius at the clamp. The results show that the effective masses of both modes drop quickly for increasing tapering. This significant reduction of the effective masses has a remarkable effect on the sensor's responsivity. Figure 4(b) shows the responsivity calculated for the first two modes. Again, we plot the ratio of the responsivity of the first two modes of a tapered NW to that of a uniform NW with the same length and same radius at the clamp. Remarkably, the responsivity for both modes increases by a factor of more than 20 for $\alpha>0.8$.

Finally, we estimate the minimum detectable mass change with a tapered NW. If we consider the minimum detectable frequency shift limited by thermomechanical fluctuations of a resonator driven at constant mean square amplitude, then $\delta M$ can be written as ${ }^{31}$

$$
\delta M=m_{n} \sqrt{\frac{E_{t h}}{E_{c}} \frac{2 B}{\pi f_{n} Q_{n}}},
$$

where $B$ is the measurement bandwidth, $Q_{n}$ is the mode quality factor, $E_{t h}=k_{B} T$ is the thermal energy and $E_{c}$ is the drive energy. Figure 4(c) shows the calculation of the ratio of the minimum detectable mass with a tapered NW to that of a

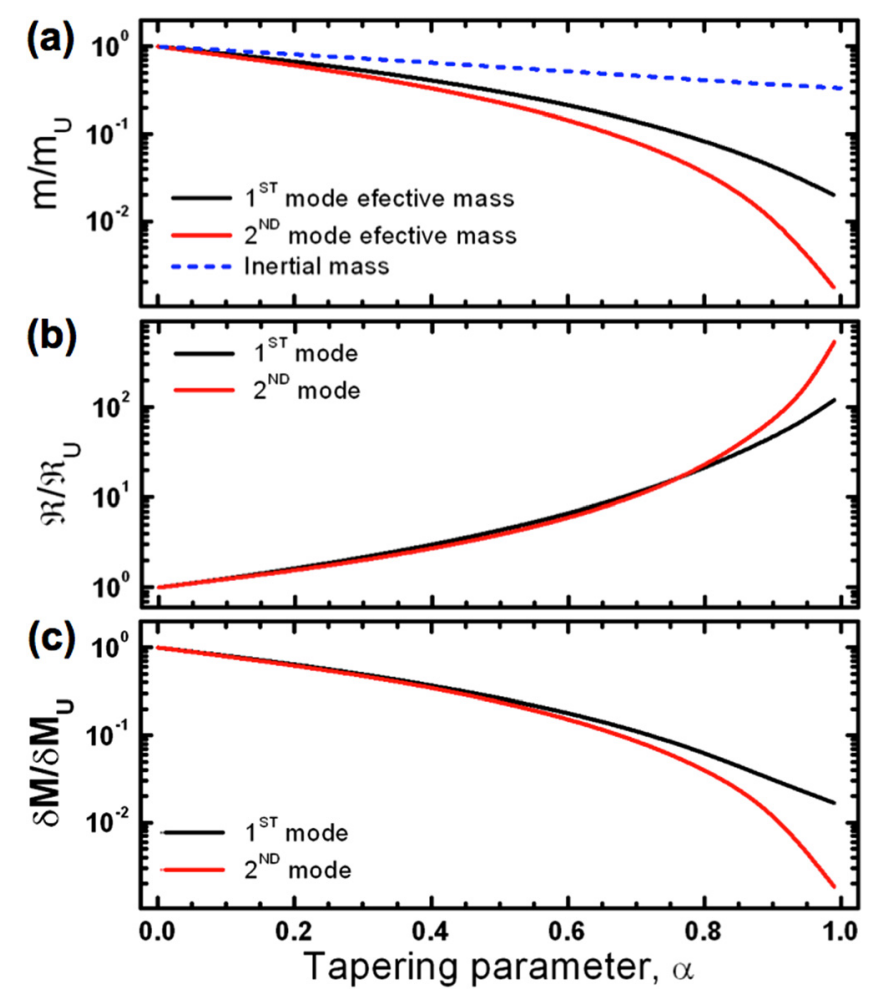

FIG. 4. (a) Ratio of the inertial mass (dashed line) and effective masses (solid lines) of the resonant modes of a tapered NW to those of a uniform one with the same length and radius at the base. (b) Ratio of the responsivity of a tapered NW to that of a uniform one with the same length and radius at the base. (c) Ratio of the minimum detectable mass with a tapered NW to that of a uniform one with the same length and radius at the base.

uniform NW with the same length and same radius at the clamp. The temperature, bandwidth, quality factor, and drive energy are considered to be equal for the two cases. The graph indicates that the minimum detectable mass by a tapered NW with $\alpha>0.8$ is more than one order of magnitude smaller for both modes. Remarkably, the ratio exceeds two orders of magnitude for the second mode and $\alpha>0.9$. We emphasize here that the physical origin of this sensitivity enhancement is not given simply by the reduction in size of a tapered NW as compared to a uniform NW. In the limit case of a conical NW with $\alpha \approx 1$, the inertial mass is reduced by a factor of $1 / 3$. However, the minimum detectable mass decreases by almost three orders of magnitude.

In conclusion, we have derived theoretical expressions for the resonance frequencies of singly clamped beam-like nanostructures as a function of their tapering degree. The theory reproduces the experimental measurements for tapered Si NWs, and it provides a general framework for the precise determination of mass and elasticity of sample adsorbates from resonance frequency shift measurements. Tapering results in localization of the vibration towards the free end, which reduces the minimum detectable mass by a conical beam-like resonator by almost two orders of magnitude for the first mode and almost tree orders of magnitude for the second mode.

This work was partially funded by projects MAT201236197, IPT-2011-0821-010000, CSD2010-00024, IOF-2009254996, and ERC-StG-2011-278860. 
${ }^{1}$ K. L. Ekinci and M. L. Roukes, Rev. Sci. Instrum. 76, 061101 (2005).

${ }^{2}$ V. Schmidt, J. V. Wittemann, S. Senz, and U. Gosele, Adv. Mater. 21, 2681 (2009).

${ }^{3}$ X. L. Feng, R. He, P. Yang, and M. L. Roukes, Nano Lett. 7, 1953 (2007).

${ }^{4}$ M. W. Li, R. B. Bhiladvala, T. J. Morrow, J. A. Sioss, K. K. Lew, J. M.

Redwing, C. D. Keating, and T. S. Mayer, Nat. Nanotechnol. 3, 88 (2008).

${ }^{5}$ M. Belov, N. J. Quitoriano, S. Sharma, W. K. Hiebert, T. I. Kamins, and S. Evoy, J. Appl. Phys. 103, 074304 (2008).

${ }^{6}$ R. He, X. L. Feng, M. L. Roukes, and P. Yang, Nano Lett. 8, 1756 (2008).

${ }^{7}$ J. M. Nichol, E. R. Hemesath, L. J. Lauhon, and R. Budakian, Appl. Phys. Lett. 93, 193110 (2008).

${ }^{8}$ B. Sanii and P. D. Ashby, Phys. Rev. Lett. 104, 147203 (2010).

${ }^{9}$ M. Sansa, M. Fernandez-Regulez, A. S. Paulo, and F. Perez-Murano, Appl. Phys. Lett. 101, 243115 (2012).

${ }^{10}$ E. Gil-Santos, D. Ramos, J. Martinez, M. Fernandez-Regulez, R. Garcia, A. San Paulo, M. Calleja, and J. Tamayo, Nat. Nanotechnol. 5, 641 (2010).

${ }^{11}$ J. M. Nichol, E. R. Hemesath, L. J. Lauhon, and R. Budakian, Phys. Rev. B 85, 054414 (2012).

${ }^{12}$ D. Ramos, E. Gil-Santos, V. Pini, J. M. Llorens, M. Fernandez-Regulez, A. San Paulo, M. Calleja, and J. Tamayo, Nano Lett. 12, 932 (2012).

${ }^{13}$ J. M. Nichol, E. R. Hemesath, L. J. Lauhon, and R. Budakian, Appl. Phys. Lett. 95, 123116 (2009).

${ }^{14}$ Y. L. Chueh, L. J. Chou, C. A. Hsu, and S. C. Kung, J. Phys. Chem. B 109, 21831 (2005).

${ }^{15}$ L. Y. Cao, B. Nabet, and J. E. Spanier, Phys. Rev. Lett. 96, 157402 (2006).

${ }^{16}$ J. Zhu, Z. F. Yu, G. F. Burkhard, C. M. Hsu, S. T. Connor, Y. Q. Xu, Q. Wang, M. McGehee, S. H. Fan, and Y. Cui, Nano Lett. 9, 279 (2009).
${ }^{17}$ J. B. Hannon, S. Kodambaka, F. M. Ross, and R. M. Tromp, Nature (London) 440, 69 (2006).

${ }^{18}$ Y. W. Wang, V. Schmidt, S. Senz, and U. Gosele, Nat. Nanotechnol. 1, 186 (2006).

${ }^{19}$ L. Y. Cao, B. Garipcan, J. S. Atchison, C. Y. Ni, B. Nabet, and J. E. Spanier, Nano Lett. 6, 1852 (2006).

${ }^{20}$ W. I. Park, G. F. Zheng, X. C. Jiang, B. Z. Tian, and C. M. Lieber, Nano Lett. 8, 3004 (2008).

${ }^{21}$ S. Krylyuk, A. V. Davydov, and I. Levin, Acs Nano 5, 656 (2011).

${ }^{22}$ A. San Paulo, J. Bokor, R. T. Howe, R. He, P. Yang, D. Gao, C. Carraro, and R. Maboudian, Appl. Phys. Lett. 87, 053111 (2005).

${ }^{23}$ M. R. Nelis, L. Yu, W. Zhang, Y. Zhao, C. Yang, A. Raman, S. Mohammadi, and J. F. Rhoads, Nanotechnology 22, 455502 (2011).

${ }^{24}$ S. Rast, C. Wattinger, U. Gysin, and E. Meyer, Rev. Sci. Instrum. 71, 2772 (2000).

${ }^{25}$ S. Abohadima and M. H. Taha, Eng. Mech. 15, 3 (2008).

${ }^{26}$ K. L. Ekinci, X. M. H. Huang, and M. L. Roukes, Appl. Phys. Lett. 84, 4469 (2004).

${ }^{27}$ K. Jensen, K. Kim, and A. Zettl, Nat. Nanotechnol. 3, 533 (2008).

${ }^{28}$ A. K. Naik, M. S. Hanay, W. K. Hiebert, X. L. Feng, and M. L. Roukes, Nat. Nanotechnol. 4, 445 (2009).

${ }^{29}$ J. Chaste, A. Eichler, J. Moser, G. Ceballos, R. Rurali, and A. Bachtold, Nat. Nanotechnol. 7, 301 (2012).

${ }^{30}$ S. Schmid, M. Kurek, J. Q. Adolphsen, and A. Boisen, Sci. Rep. 3, 1288 (2013).

${ }^{31}$ K. L. Ekinci, Y. T. Yang, and M. L. Roukes, J. Appl. Phys. 95, 2682 (2004).

${ }^{32}$ See supplementary material at http://dx.doi.org/10.1063/1.4813819 for details about Si NW growth conditions and theoretical modeling. 